\title{
Thrombopoietin expands hematopoietic stem cells after transplantation
}

\author{
Norma Fox, ${ }^{1}$ Greg Priestley, ${ }^{2}$ Thalia Papayannopoulou, ${ }^{2}$ and Kenneth Kaushansky ${ }^{1}$ \\ ${ }^{1}$ University of California San Diego School of Medicine, Department of Medicine, San Diego, California, USA \\ ${ }^{2}$ University of Washington School of Medicine, Division of Hematology, Seattle, Washington, USA
}

\begin{abstract}
Multiple lines of evidence indicate that thrombopoietin (TPO) contributes to the development of hematopoietic stem cells (HSC), supporting their survival and proliferation in vitro. To determine whether TPO supports the impressive expansion of HSC observed following transplantation, we transplanted normal marrow cells into lethally irradiated $\mathrm{Tpo}^{-/-}$and $\mathrm{TpO}^{+/+}$mice and quantified HSC self-renewal and expansion and hematopoietic progenitor cell homing. Although essentially identical numbers of marrow-associated colony forming unit-culture (a surrogate measure of stem cell homing) were observed in each type of recipient 24 hours following transplantation, we found that a minimum of fourfold greater numbers of marrow cells were required to radioprotect $T$ po-null mice than to radioprotect controls. To assess whether long-term repopulating (LTR) HSCs self-renew and expand in $\mathrm{TpO}^{-/-}$recipients or controls, we performed limiting-dilution secondary transplants using donor cells from the $\mathrm{Tpo}^{-/-}$or $T \mathrm{To}^{+/+}$recipients 5-7.5 weeks following primary transplantation. We found that LTR HSCs expand to levels 10-20 times greater within this time period in normal recipients than in Tpo-null mice and that physiologically relevant amounts of TPO administered to the $T_{p o^{-/-}}$recipients could substantially correct this defect. Our results establish that TPO greatly promotes the self-renewal and expansion of HSCs in vivo following marrow transplantation.
\end{abstract}

J. Clin. Invest. 110:389-394 (2002). doi:10.1172/JCI200215430.

\section{Introduction}

Thrombopoietin (TPO) was first cloned and its recombinant protein first produced and characterized in 1994 (1-4). Initial studies with the recombinant protein were directed toward demonstrating that the cytokine was the primary regulator of megakaryopoiesis and platelet production. In this regard it was rapidly appreciated that the cloned gene fulfilled all of the criteria historically set out for it $(5,6)$. For example, TPO affects nearly every stage of megakaryocyte (MK) development from the committed progenitor to the mature platelet. The hormone is the most potent cytokine to stimulate the development of MK colonies from marrow progenitors in semisolid cultures (7), and it induces the expression of glycoprotein IIb/IIIa, the platelet fibrinogen receptor, and the glycoprotein Ib/IX complex, the platelet vWF receptor, in immature MKs (5). In the presence of the cytokine, MKs grow in size,

Received for publication March 11, 2002, and accepted in revised form June 11, 2002.

Address correspondence to: Kenneth Kaushansky, University of California San Diego School of Medicine, Department of Medicine, 402 Dickinson Street, Suite 380, San Diego, California 92103-8811, USA. Phone: (619) 543-6170; Fax: (619) 543-3931; E-mail: kkaushansky@ucsd.edu.

Conflict of interest: No conflict of interest has been declared. Nonstandard abbreviations used: thrombopoietin (TPO); megakaryocyte (MK); hematopoietic stem cell (HSC); long-term repopulating (LTR); colony forming unit-culture (CFU-C); colony forming unit-granulocyte macrophage (CFU-GM); burst forming unit-erythroid (BFU-E). develop several types of specific granules, and form demarcation membranes, a precursor of platelet formation. And although TPO is not essential for the last stages of platelet formation (8), it does prime platelets to respond to subthreshold levels of classic agonists such as ADP, collagen, or thrombin $(9,10)$.

Based on results using partially purified preparations of the hormone, previous reports concluded that TPO is a specific growth factor for the MK and platelet lineage $(11,12)$. However, several lines of evidence using recombinant protein now indicate that the hormone exerts a profound influence on hematopoietic stem cells (HSCs). Using highly purified populations of marrow cells, two groups reported that TPO enhances the survival of murine HSCs in vitro and, in the presence of additional cytokines, could augment their proliferation $(13,14)$; this conclusion was quickly expanded to human cells (15). One of the first indications that these effects extended to in vivo hematopoiesis came with the discovery that TPO accelerates the recovery of all hematopoietic lineages following myelosuppressive therapies $(16,17)$. Moreover, mice genetically deficient in the TPO receptor, c-Mpl, display reduced levels of all types of hematopoietic progenitors (18), again suggesting the panhematopoietic effect of the cytokine. The profound effect of TPO on HSCs was then established when it was shown that all of the long-term repopulating (LTR) HSCs display $\mathrm{c}-\mathrm{Mpl}$, and that $c-m p l$-null mice display substantial reductions in the numbers of LTR HSCs $(19,20)$. This physiology was 
very recently extended to humans when it was discovered that many children with congenital amegakaryocytic thrombocytopenia, the majority of whom develop aplastic anemia within a few years of birth, display many different missense and nonsense mutations in the c-Mpl receptor (21).

Although experimental hematologists have tested several approaches to expand adult HSCs in vitro, there is little reproducible evidence that such strategies have been successful. In contrast, if a limited number of stem cells are transplanted into a lethally irradiated animal, within a few months the number of stem cells in the marrow and spleen of the recipient is in great excess of input numbers. For example, in one report of serial transplantation experiments, about ten times more LTR HSCs were found with each successive transfer of cells (22). Given the accumulating data indicating a role for TPO in stem cell biology, in the present work we sought to test whether the hormone is important for the selfrenewal and expansion of HSCs that follow marrow transplantation. We report that, for both short-term radioprotection and long-term repopulation, TPO is vital, enhancing survival following lethal irradiation at least fivefold compared with that seen in the absence of the hormone, and accelerating the renewal and repopulation of the marrow HSC pool 13- to 20-fold in the first 2 months following transplantation.

\section{Methods}

Mice and animal care. All mice used in this study were C57BL/6. The initial stem cell donors were CD 45.1 and were obtained from Taconic (Germantown, New York, USA); all others were of the CD45.2 genotype (The Jackson Laboratory, Bar Harbor, Maine, USA). TPOnull mice bred onto the C57BL/6 background were kindly provided by Fred de Sauvage (Genentech Inc., South San Francisco, California, USA) and have been previously described (23). Mice were housed in a specific pathogen-free environment, and the Animal Care Committee of the University of Washington approved all protocols involving mice.

Reagents. Recombinant murine TPO was kindly provided by Akihiro Shimosaka (Kirin Pharmaceuticals Inc., Gumma, Japan). It was administered to some recipient animals by subcutaneous injection once before transplantation and then thrice weekly for a month at a dose of $80 \mathrm{ng}$, a dose chosen based on preliminary experiments in which the capacity to restore a normal platelet count in $\mathrm{TpO}^{-/-}$mice was evaluated.

Marrow cell transplantation. Marrow cell transplantation was performed using standard protocols; mice were subjected to $1150 \mathrm{cGy}$ whole-body irradiation from a ${ }^{137} \mathrm{Cs}$ source, and then infused with donor cells by tail vein injection. Mice were fed standard mouse chow, and antibiotics were administered in the drinking water for 1 week prior to and 3 weeks following transplantation. For secondary transplants, 5-7.5 weeks following primary marrow cell infusion, mice were sacrificed; then single-cell suspensions of femoral,
Table 1

Radioprotection and short-term repopulation in $\mathrm{TpO}^{-/-}$mice

\begin{tabular}{|c|c|c|c|c|}
\hline Recipient & $\begin{array}{l}\text { Donor } \\
\text { cell no. }\end{array}$ & $n$ & $\begin{array}{l}\text { \% Survival } \\
\text { (weeks) }\end{array}$ & $\begin{array}{c}\% \text { CD } 45.1^{+} \\
\text {following recovery }\end{array}$ \\
\hline \multicolumn{5}{|l|}{ Experiment 1} \\
\hline $\mathrm{Tpo}^{+/+}$ & $2 \times 10^{5}$ & 10 & $90(7.5)$ & 86 \\
\hline Tpo $o^{-/-}$ & $2 \times 10^{5}$ & 10 & $20(7.5)$ & 80 \\
\hline Tpo-1- & $1 \times 10^{6}$ & 10 & $80(7.5)$ & 85 \\
\hline \multicolumn{5}{|l|}{ Experiment 2} \\
\hline$T p o^{+/+}$ & $1 \times 10^{6}$ & 4 & $100(5)$ & ND \\
\hline Tpo $o^{-/-}$ & $1 \times 10^{6}$ & 4 & $25(5)$ & ND \\
\hline Tpo $o^{-/-}(+\mathrm{TPO})$ & $1 \times 10^{6}$ & 4 & $25(5)$ & ND \\
\hline
\end{tabular}

Normal or $\mathrm{Tpo}^{-/-}$recipients were infused with the indicated number of CD $45.1^{+}$ marrow cells, and survival and percent $C D 45.1$ positivity were determined at the indicated number of weeks. ND, not determined; +TPO, mice were administered $80 \mathrm{ng}$ recombinant TPO twice to thrice weekly until sacrifice.

tibial, and pelvic marrow were prepared and infused into normal CD $45.2^{+}$recipients that were treated with antibiotics for 1 week prior to and 3 weeks following transplantation. Blood was sampled for CD45 analysis at 12 and 22 weeks; a minimum of four mice at each time point were analyzed, and the results are reported as mean percentage $\mathrm{CD} 45.2^{+}$cells \pm SEM.

Marrow and spleen colony-forming cell homing experiments. Donor mice were sacrificed by cervical dislocation and marrow cells prepared. Three groups of recipient mice were irradiated with $1150 \mathrm{cGy}$ and infused with marrow cells containing one of two doses of colony forming unit-culture (CFU-C). One day later the mice were sacrificed and single-cell suspensions of marrow and spleen were prepared and cultured in triplicate for colony forming unit-granulocyte macrophage (CFU-GM), CFU-Mix, and burst forming unit-erythroid (BFU-E) by standard methods (16). The total number of CFU-C recovered after 24 hours was expressed as a percentage of infused CFU-C. In a second test of the capacity of marrow cells to home, we labeled cells for 10 minutes at $37^{\circ} \mathrm{C}$ in $0.5 \mu \mathrm{M} \mathrm{5,6}$ carboxyfluorescein diacetate succinimidyl ester (CFSE), washed and infused them into wild-type and $\mathrm{TpO}^{-/-}$mice, and then evaluated marrow of the recipients for $\mathrm{CFSE}^{+}$cells 24 hours later.

Flow cytometry analysis. To determine the origin of the circulating hematopoietic cells following transplantation, blood was obtained from transplanted mice 3 and 5 months following the procedure and analyzed by flow cytometry using a phycoerythrin-labeled $\mathrm{mAb}$ to murine CD45.1 (Pharmingen, La Jolla, California, USA).

\section{Results}

TPO enhances the capacity of transplanted marrow cells to radioprotect. In order to determine whether TPO was important for the ability of short-term repopulating stem cells to radioprotect recipient mice, we transplanted $2 \times 10^{5}$ normal CD45.1 marrow cells into normal CD45.2 recipients and compared post-transplant survival to that of CD45.2 $\mathrm{Tpo}^{-/-}$mice transplanted with either $2 \times 10^{5}$ or $1 \times 10^{6}$ normal CD45.1 marrow cells. As shown in Table 1, we found that nine of ten 
Table 2

Marrow and splenic homing of normal marrow cells in $\mathrm{Tpo}^{-/-}$mice

Recipient

No. of
FU-C infused

$\%$ Marrow

recovery

$\%$ Splenic recovery

Experiment 1, day 1

$\mathrm{TpO}^{+/+}$

$1.8 \times 10^{5}$

5

$10.9 \pm 0.25$

$3.0 \pm 0.17$

$1.8 \times 10^{5}$

5

$9.7 \pm 0.60$

$4.9 \pm 0.86$

Experiment 2, day 1

$\mathrm{TpO}^{+/+}$

$6.3 \times 10^{4}$

$17 \pm 4.2$

$5.3 \pm 1.3$

$6.3 \times 10^{4}$

$19 \pm 2.3$

$4.9 \pm 0.60$

Marrow cells containing a known number of CFU-C were infused into lethally irradiated $\mathrm{TpO}^{+/+}$or $\mathrm{TpO}^{-/-}$recipients, and the mice were sacrificed 24 hours later. The results represent the mean number of CFU-C $( \pm$ SEM) recovered from the marrow or the spleen of the recipients, based on triplicate cultures for CFU-GM, CFU-Mix, and BFU-E colonies in each mouse, then expressed as the percent of CFU-C injected based on a single femur representing $6 \%$ of the total marrow of the animal. There were no statistically significant differences between any of the groups.

normal recipients of $2 \times 10^{5}$ normal marrow cells survived; a mean of $86 \%$ of the hematopoietic cells were CD $45.1^{+}$in the recipients. In contrast, only two of ten $\mathrm{TPO}^{-/-}$recipients of the same number of normal marrow cells survived, with $80 \%$ of those hematopoietic cells CD $45.1^{+}$. The infusion of $1 \times 10^{6}$ normal marrow cells was required to rescue eight of ten transplanted $\mathrm{Tpo}^{-/-}$mice. In a second experiment, $1 \times 10^{6}$ normal marrow cells were injected into four $\mathrm{Tpo}^{+/+}$recipients, four $\mathrm{Tpo}^{-/-}$recipients that were given saline thrice weekly, and four $T \mathrm{po}^{-/-}$recipients given thrice-weekly injections of $80 \mathrm{ng}$ recombinant murine TPO. This dose and dosing schedule of TPO was determined to be the most physiologically relevant, as it returned the platelet count of $\mathrm{TpO}^{-/-}$mice to normal levels and maintained it at those levels. All four of the $T \mathrm{po}^{+/+}$ recipients survived more than 5 weeks; in contrast, only one of four of both $\mathrm{Tpo}^{-/-}$recipient groups survived more than 5 weeks. Thus, since we saw little or no radiation-induced death in the control groups, the four- to fivefold enhanced radioprotection afforded by normal marrow cells in lethally irradiated normal mice compared with $\mathrm{TpO}^{-/-}$mice is a minimal estimation.

TPO does not affect the homing of hematopoietic cells to marrow or spleen. The previous results assessing radioprotection and short-term repopulation could have been due either to reduced stem cell homing to marrow in $\mathrm{TPO}^{-/-}$mice or to defective expansion of the stem cells lodged in the $\mathrm{TpO}^{-/-}$marrow. To distinguish between these possibilities, in two sets of experiments we transplanted normal marrow containing either $1.8 \times 10^{5}$ or $6.3 \times 10^{4}$ CFU-C into groups of three to five lethally irradiated $\mathrm{TpO}^{-/-}$or normal mice and quantitated CFU-C recovered in the marrow 24 hours later. Previous studies have indicated that CFU-C homing is an accurate surrogate for stem cell homing (24-26). As shown in Table 2, we found that in both sets of experiments cells homed as efficiently in $\mathrm{TpO}^{-/-}$recipients as in wild-type mice, the values in $\mathrm{TpO}^{-/-}$mice being $89 \%$ and $112 \%$ of normal for homing to the marrow, and $163 \%$ and $94 \%$ of normal for lodgment in the spleen, respectively. In a second type of analysis, we labeled normal donor marrow cells with the membrane-soluble dye CFSE and assessed the percentage of marrow cells that were $\mathrm{CFSE}^{+}$ 24 hours following their infusion into lethally irradiated recipients. We found a mean of $1.4 \% \mathrm{CFSE}^{+}$marrow cells in $\mathrm{Tpo}^{+/+}$recipients and $1.3 \% \mathrm{CFSE}^{+}$in the $\mathrm{Tpo}^{-/-}$ recipients. Thus, the difference in radioprotection and short-term repopulation following transplantation of normal marrow cells into $\mathrm{Tpo}^{+/+}$and $\mathrm{TpO}^{-/-}$mice was not due to abnormal hematopoietic cell homing in the latter group of mice.

TPO enhances the self-renewal and expansion of LTR HSCs. Previous studies demonstrated that upon infusion into lethally irradiated mice, HSCs expand within the first few months following the procedure. To determine whether TPO affects this aspect of stem cell physiology and to quantitate the effect, limiting-dilution secondary transplants were performed using marrow cells from the $T \mathrm{Po}^{+/+}$or $\mathrm{Tpo}^{-/-}$primary transplant recipients from the two experiments reported upon in Table 1, 5 or 7.5 weeks following primary transplantation. In the first experiment, the $\mathrm{TPO}^{+/+}$and $\mathrm{TPO}^{-/-}$mice transplanted with $2 \times 10^{5}$ normal marrow cells 7.5 weeks earlier were sacrificed, and $3 \times 10^{5}$ marrow cells from the normal primary transplant recipients and either $1 \times 10^{6}$ or $2.5 \times 10^{6}$ marrow cells from the $\mathrm{Tpo}^{-/-}$ primary transplant recipients were infused into normal, lethally irradiated C57BL/6 secondary recipients. In the second experiment, the primary transplant recipients were sacrificed at 5 weeks, and limiting-dilution secondary transplants were performed to calculate HSC expansion during the primary transplant. In the second experiment we also studied whether the administration of TPO during the primary transplant period could correct for the genetic deficiency. The survival curves from the first set of secondary transplants are shown in Figure 1, and the results from both

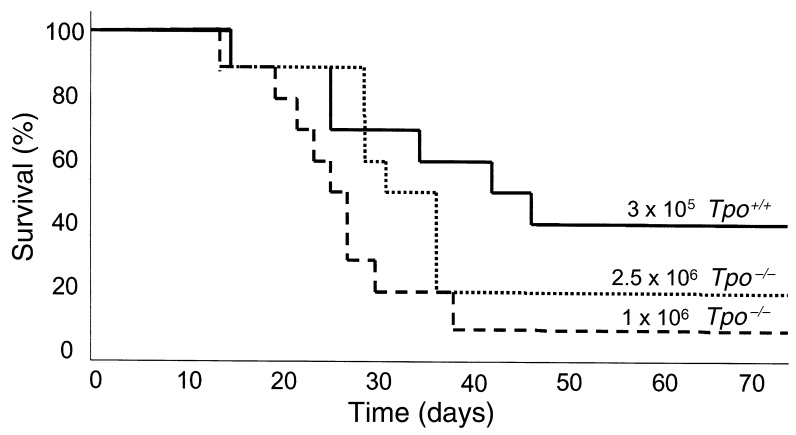

\section{Figure 1}

Survival of mice transplanted with marrow cells from previously transplanted $\mathrm{TpO}^{+/+}$or $\mathrm{TpO}^{-/-}$mice. The curves shown are survival as a function of time for: normal recipient mice transplanted with $3 \times 10^{5}$ marrow cells from normal recipient mice transplanted 7.5 weeks earlier with $2 \times 10^{5}$ normal marrow cells (solid line); $1 \times 10^{6}$ marrow cells from $\mathrm{TpO}^{-/-}$mice transplanted 7.5 weeks earlier with $2 \times 10^{5}$ normal marrow cells (dashed line); and $2.5 \times 10^{6}$ marrow cells from $\mathrm{TpO}^{-/-}$mice transplanted 7.5 weeks earlier with $2 \times 10^{5}$ normal marrow cells (dotted line). 
Table 3

Secondary transplantation from $\mathrm{TpO}^{+/+}$and $\mathrm{Tpo}^{-/-}$primary transplant recipients

\begin{tabular}{|c|c|c|c|c|c|c|}
\hline Donor & $\begin{array}{c}\text { (No. of cells } \\
\text { in } 1^{\circ} \text { ) }\end{array}$ & $\begin{array}{c}\text { Time of } 2^{\circ} \\
\text { (wks) }\end{array}$ & $\begin{array}{l}\text { No. of cells } \\
\text { in } 2^{\circ}\end{array}$ & $\begin{array}{l}\text { No. of } \\
\text { recipients }\end{array}$ & \% Survival & $\begin{array}{c}\text { Relative } \\
\text { expansion }\end{array}$ \\
\hline \multicolumn{7}{|l|}{ Experiment 1} \\
\hline $\begin{array}{l}\text { Tpo } o^{+/+} \\
T p o^{-/-} \\
T p o^{-/-}\end{array}$ & $\begin{array}{l}\left(2 \times 10^{5}\right) \\
\left(2 \times 10^{5}\right) \\
\left(2 \times 10^{5}\right)\end{array}$ & $\begin{array}{l}7.5 \\
7.5 \\
7.5\end{array}$ & $\begin{array}{r}3 \times 10^{5} \\
1 \times 10^{6} \\
2.5 \times 10^{6}\end{array}$ & $\begin{array}{l}10 \\
10 \\
10\end{array}$ & $\begin{array}{l}40 \\
10 \\
20\end{array}$ & $\begin{array}{l}(1.0) \\
0.076 \\
0.060\end{array}$ \\
\hline \multicolumn{7}{|l|}{ Experiment 2} \\
\hline $\begin{array}{l}T p o^{+/+} \\
T p o^{-/-} \\
T p o^{-1-}+\text { TPO } \\
T p o^{-/-}+\text {TPO }\end{array}$ & $\begin{array}{l}\left(1 \times 10^{6}\right) \\
\left(1 \times 10^{6}\right) \\
\left(1 \times 10^{6}\right) \\
\left(1 \times 10^{6}\right)\end{array}$ & $\begin{array}{l}5 \\
5 \\
5 \\
5\end{array}$ & $\begin{array}{r}5 \times 10^{5} \\
7.5 \times 10^{6} \\
1 \times 10^{6} \\
7.5 \times 10^{6}\end{array}$ & $\begin{array}{l}10 \\
10 \\
10 \\
10\end{array}$ & $\begin{array}{c}100 \\
70 \\
80 \\
100\end{array}$ & $\begin{array}{c}(1.0) \\
0.048 \\
0.40 \\
\mathrm{NC}\end{array}$ \\
\hline
\end{tabular}

Mice having received primary transplants 5-7.5 weeks earlier were sacrificed, and limiting dilutions of marrow cells were infused into $\mathrm{C} 57 \mathrm{BL} / 6$ recipients and their survival monitored. The inocula were composed of marrow cells mixed from multiple donor mice, except in the $\mathrm{TpO}^{-/-}$and $\mathrm{TpO}^{-/-}+$TPO groups in experiment 2 , in which only a single primary transplant recipient of each group had survived to serve as a secondary transplant donor. At 5 months, all of the surviving mice displayed a majority of CD $45.1^{+}$hematopoietic cells in the peripheral blood. The relative expansion of HSCs in the 5-7.5 weeks in the primary transplant recipients was calculated based on the number of cells infused into the secondary recipients and the relative survival of the secondary transplant recipients. The calculation of relative expansion in the $\mathrm{TpO}^{-/-}$and $\mathrm{TpO}^{-I_{-}^{-}+\mathrm{TPO}}$ low-cell dose groups in experiment 2 is a minimal estimate, as there were no deaths in the control $T p 0^{+/+}$ group. NC, non-calculable, since there were no deaths in either the $\mathrm{TpO}^{+/+}$or the high-cell dose $\mathrm{TpO}^{-/-}+\mathrm{TPO}$ groups. $1^{\circ}$, primary; $2^{\circ}$, secondary.

experiments are tabulated and the relative stem cell expansion calculated in Table 3

If one assumes that the successful re-engraftment of, for example, four of ten mice represents the presence of twice as many HSCs in the transplant inoculum as if two of ten mice were engrafted, then one can calculate the relative numbers of stem cells present in the primary transplant recipients. As illustrated in Table 3 , the number of HSCs present in the marrow of mice transplanted 5 weeks earlier with normal HSCs was approximately 20 -fold greater when the transplantation inoculum was derived from transplanted normal mice than when the transplanted cells had come from $\mathrm{TpO}^{-/-}$mice transplanted with normal HSCs. At 7.5 weeks, there were 13- to 16-fold more HSCs in the normal primary recipients than in their $\mathrm{Tpo}^{-/-}$counterparts. Flow cytometric analysis for CD45.1 indicated that $81 \% \pm 2 \%$ (mean \pm SEM) of peripheral blood cells were of donor type 12 weeks after transplantation, and $76 \% \pm 5.5 \%$ were of donor origin 22 weeks after secondary transplantation, demonstrating that hematological recovery was due to the transplanted cells, not to endogenous $\left(\mathrm{CD} 45.2^{+}\right)$recovery. As seen in the second experiment, the administration of a small dose of recombinant TPO to the $T \mathrm{TO}^{-/-}$recipients substantially corrected (i.e., to $40 \%$ of normal) the defect in HSC expansion seen in the null mice.

\section{Discussion}

The purpose of the present study was to determine whether the effects of TPO on steady-state numbers of HSCs also extend to the self-renewal and early expansion that these cells undergo following marrow transplantation. If so, then the use of the recombinant protein following marrow or stem cell transplantation, especially in recipients in which limiting numbers of cells are available, such as in patients who are difficult to mobilize, or from small-volume cord blood transplant products, might prove of therapeutic value. Although we didn't test that hypothesis directly in this study, our results clearly indicate that TPO plays an important role in the initial stem cell renewal and expansion seen in mice transplanted with limiting numbers of HSCs.

In the initial stage of each experiment, the level of radioprotection provided by the transplantation of the normal $\mathrm{HSCs}$ in $\mathrm{TpO}^{+/+}$and $\mathrm{Tpo}^{-/-}$recipient mice was compared; in both experiments, survival in the wild-type recipient was improved four to five times over survival in null mice. It is possible that this is an underestimate of the Tpo effect, as the survival in the control arm was $90 \%$ and $100 \%$ in the two experiments, indicating that there might have been even more short-term repopulating activity in the $2 \times 10^{5}$ or $1 \times 10^{6}$ cell inoculum measurable in the wildtype recipients. Additional experiments using lower cell doses could sort this issue out.

The improved survival afforded by the TPO-replete host could have been due to an enhanced capacity of HSCs to home to and lodge in the marrow microenvironment rather than to any favorable effect of the hormone on the initial survival or proliferation of the short-term repopulating cells that provide radioprotection. Although stromal cells are not thought to be targets of TPO, it is clear that endothelial cells express the TPO receptor $\mathrm{c}-\mathrm{Mpl}$ (27). Along with potential TPO effects on stem cells while they traffic in the circulation, these considerations raise the theoretical possibility that HSC homing might be affected in the Tpo-null mouse. Thus, to be certain that the effects of TPO were on the self-renewal and expansion of HSCs, we injected marrow cells containing two different numbers of CFU-C into normal and Tpo-null mice and determined the number of such progenitors that lodged in the primary hematopoietic organs of mice 24 hours later. We chose this time point to avoid confusing the effects of CFU-C lodging and the proliferation of more primitive, CFU-C-generating progenitors that had homed to the marrow or spleen. We found virtually identical numbers of CFU-C in the marrow and spleens of the recipients, strongly suggesting that differential homing did not account for our radioprotection results.

We next studied whether TPO affected expansion of LTR HSCs by performing limiting-dilution secondary transplant experiments, using our primary $\mathrm{Tpo}^{+/+}$and $\mathrm{TpO}^{-/-}$recipients as donors. We found a profound reduction in the capacity of HSCs to expand in $\mathrm{TpO}^{-/-}$ 
recipients, from a minimum of 13 -fold to a maximum of 21-fold less expansion than in the wild-type mice. Although in one experiment $100 \%$ survival in the $\mathrm{TpO}^{+/+}$control group made it impossible to gauge whether the difference in relative HSC expansion might have been even greater if we had used a smaller inoculum of cells, we doubt that this is the case, as the two experiments yielded quite similar levels of LTR HSC expansion 5 and 7.5 weeks following primary transplantation. Studies performed by Iscove and Nawa demonstrated that serial transplantation experiments of the type performed in this report lead to about tenfold increases in LTR HSC numbers over input levels (22), suggesting that TPO might account for the maximal level of stem cell expansion possible under these conditions. Moreover, experiments performed by Eaves's group indicate that genetic alteration of the c-Kit/Steel Factor system imposes a similar level of HSC deficiency (28). In that study, transplantation of $W^{41} / W^{41}$ stem cells into normal recipients reduced HSC expansion 17-fold compared with wild-type donor cells. Although the $W^{41}$ allele is a hypomorph and not a complete null, taken together the similar quantitative analyses suggest that TPO and Steel Factor might act synergistically on expansion of HSCs in vivo, as they do in vitro $(7,13,14)$.

In the second set of our experiments we also tested whether exogenous TPO could correct the HSC expansion defect seen in Tpo-null mice. We first tested for the amount of TPO needed to provide a "replacement dose" for Tpo-null mice, as the use of pharmacological concentrations of a hormone might exert effects not seen with the physiological level. By administering different doses of recombinant TPO to Tpo-null mice, we found in preliminary experiments that $80 \mathrm{ng}$ administered thrice weekly led to normalization of the platelet count after about 1 month. Using this dose of TPO in $\mathrm{TpO}^{-/-}$recipients, we found that HSCs expand about $40 \%$ as well as in wild-type recipients. However, like the quantitative considerations raised above, this calculation is the maximal correction that could have occurred in this experiment, as all the control animals survived and repopulated. It is possible that if we had used lower numbers of marrow cells for the secondary transplants we might have seen a less impressive normalization of HSC expansion. This argument is of more than academic interest: if exogenous TPO administered to Tponull mice does not fully correct the stem cell defect of these animals, it is possible that the local effect of stromal cell-derived hormone is greater than that coming from the circulation. In this regard, debate remains in the field as to whether marrow stromal cell production of TPO is of importance for stress hematopoiesis.

Based on the effect of TPO on HSC self-renewal and expansion, one might predict that the administration of exogenous TPO to transplant recipients would enhance engraftment kinetics. In fact, this has been tested in both preclinical and clinical settings, and the results have been disappointing. For example, although the administration of TPO to murine stem cell transplant recipients was reported to accelerate hematological recovery in one study, the effect was quite modest (29) and did not occur in another model of marrow transplantation if TPO was given only to the recipient (30). Similarly disappointing results were reported following peripheral blood HSC transplantation into patients (31), although a modest effect was seen following the use of bone marrow stem cells (32). Moreover, we found that the administration of TPO in the short term, during the primary transplant experiments illustrated in Table 1, failed to radioprotect $\mathrm{TpO}^{-/-}$mice given a standard number of marrow cells. These results do not necessarily cast doubt on the conclusions of the present study, as even the levels of TPO that are normally present in mice were sufficient to at least partially correct the LTR HSC expansion defect in $\mathrm{TpO}^{-/-}$mice when administered exogenously in our experiments. Moreover, given the higher-than-normal levels of TPO that occur in animals and patients following myelosuppressive therapy, such as the conditioning therapy for transplantation, it is very likely that endogenous TPO is sufficient to provide for HSC self-renewal and expansion following transplantation. In our studies we failed to find an effect of exogenous TPO on shortterm radioprotection, in contrast to the results with LTR HSCs. It is possible that the effect of TPO on stem cell expansion requires more time than is required to radioprotect a mouse, or that a minor effect could have been revealed if additional dose-finding experiments had been performed. Thus, whether exogenously administered TPO is useful for clinical transplantation will require additional study.

Finally, although we use the term "expansion" throughout this report to indicate that the numbers of HSCs found 5 or 7.5 weeks following transplantation are greater than the numbers of input cells, it is possible that TPO affects both HSC survival and proliferation, or that it only acts on the former. In this regard, using p53-null mice, Pestina and colleagues recently reported that TPO accelerates blood cell recovery following the administration of otherwise lethal chemoradiotherapy by preventing programmed cell death of hematopoietic cells (33). As recovery from such toxic insults is thought to be dependent on primitive marrow hematopoietic cells, it is possible that the effect of TPO on HSC expansion might be dependent on the same effect. The relative importance of these two fundamental processes on TPOinduced HSC expansion following transplantation deserves further study.

\section{Acknowledgments}

The authors wish to acknowledge the support of the NIH (grants R01 CA31615, R01 DK44855, and R01 HL58734).

\footnotetext{
1. de Sauvage, F.J., et al. 1994. Stimulation of megakaryocytopoiesis and thrombopoiesis by the c-Mpl ligand. Nature. 369:533-538.

2. Lok, S., et al. 1994. Cloning and expression of murine thrombopoietin
} 
cDNA and stimulation of platelet production in vivo. Nature. 369:565-568.

3. Bartley, T.D., et al. 1994. Identification and cloning of a megakaryocyte growth and development factor that is a ligand for the cytokine receptor Mpl. Cell. 77:1117-1124.

4. Sohma, Y., et al. 1994. Molecular cloning and chromosomal localization of the human thrombopoietin gene. FEBS Lett. 353:57-61.

5. Kaushansky, K., et al. 1994. Promotion of megakaryocyte progenitor expansion and differentiation by the c-Mpl ligand thrombopoietin. Nature. 369:568-571.

6. Kaushansky, K. 1995. Thrombopoietin: the primary regulator of platelet production. Blood. 86:419-431.

7. Broudy, V.C., Lin, N.L., and Kaushansky, K. 1995. Thrombopoietin (c-mpl ligand) acts synergistically with erythropoietin, stem cell factor, and IL-11 to enhance murine megakaryocyte colony growth and increases megakaryocyte ploidy in vitro. Blood. 85:1719-1726.

8. Choi, E.S., Nichol, J.L., Hokom, M.M., Hornkohl, A.C., and Hunt, P. 1995. Platelets generated in vitro from proplatelet-displaying human megakaryocytes are functional. Blood. 85:402-413.

9. Chen, J., Herceg-Harjacek, L., Groopman, J.E., and Grabarek, J. 1995. Regulation of platelet activation in vitro by the c-Mpl ligand, thrombopoietin. Blood. 86:4054-4062.

10. Kojima, H., et al. 1995. Modulation of platelet activation in vitro by thrombopoietin. Thromb. Haemost. 74:1541-1545.

11. Hill, R.J., and Levin, J. 1989. Regulators of thrombopoiesis: their biochemistry and physiology. Blood Cells. 15:141-166.

12. McDonald, T.P. 1988. Thrombopoietin: its biology, purification, and characterization. Exp. Hematol. 16:201-205.

13. Ku, H., Yonemura, Y., Kaushansky, K., and Ogawa, M. 1996. Thrombopoietin, the ligand for the $\mathrm{Mpl}$ receptor, synergizes with steel factor and other early-acting cytokines in supporting proliferation of primitive hematopoietic progenitors of mice. Blood. 87:4544-4551.

14. Sitnicka, E., et al. 1996. The effect of thrombopoietin on the proliferation and differentiation of murine hematopoietic stem cells. Blood. 87:4998-5005

15. Kobayashi, M., Laver, J.H., Kato, T., Miyazaki, H., and Ogawa, M. 1996. Thrombopoietin supports proliferation of human primitive hematopoietic cells in synergy with steel factor and/or interleukin-3. Blood. 88:429-436.

16. Kaushansky, K., et al. 1995. Thrombopoietin expands erythroid progenitors, increases red cell production, and enhances erythroid recovery after myelosuppressive therapy. J. Clin. Invest. 96:1683-1687.

17. Neelis, K.J., et al. 1997. Prevention of thrombocytopenia by thrombopoietin in myelosuppressed rhesus monkeys accompanied by prominent erythropoietic stimulation and iron depletion. Blood. 90:58-63.

18. Alexander, W.S., Roberts, A.W., Nicola, N.A., Li, R., and Metcalf, D. 1996 Deficiencies in progenitor cells of multiple hematopoietic lineages and defective megakaryocytopoiesis in mice lacking the thrombopoietin receptor c-Mpl. Blood. 87:2162-2170.

19. Kimura, S., Roberts, A.W., Metcalf, D., and Alexander, W.S. 1998. Hematopoietic stem cell deficiencies in mice lacking c-Mpl, the receptor for thrombopoietin. Proc. Natl. Acad. Sci. USA. 95:1195-1200.

20. Solar, G.P., et al. 1998. Role of c-mpl in early hematopoiesis. Blood. 92:4-10.

21. Ballmaier, M., et al. 2001. c-mpl mutations are the cause of congenital amegakaryocytic thrombocytopenia. Blood. 97:139-146.

22. Iscove, N.N., and Nawa, K. 1997. Hematopoietic stem cells expand during serial transplantation in vivo without apparent exhaustion. Curr. Biol. 7:805-808.

23. Moore, M.W., and deSauvage, F.J. 1996. Low levels of erythroid and myeloid progenitors in thrombopoietin- and $c$-mpl-deficient mice. Blood. 88:803-808.

24. Papayannopoulou, T., and Craddock, C. 1997. Homing and trafficking of hemopoietic progenitor cells. Acta Haematol. 97:97-104.

25. Cui, J., et al. 1999. Bone marrow cell trafficking following intravenous administration. Br. J. Haematol. 107:895-902.

26. Oostendorp, R.A., Ghaffari, S., and Eaves, C.J. 2000. Kinetics of in vivo homing and recruitment into cycle of hematopoietic cells are organ-specific but CD44-independent. Bone Marrow Transplant. 26:559-566.

27. Vigon, I., et al. 1992. Molecular cloning and characterization of $M P L$, the human homolog of the v-mpl oncogene: identification of a member of the hematopoietic growth factor receptor superfamily. Proc. Natl. Acad. Sci. USA. 89:5640-5644.

28. Miller, C.L., et al. 1996. Studies of W mutant mice provide evidence for alternate mechanisms capable of activating hematopoietic stem cells. Exp. Hematol. 24:185-194.

29. Molineux, G., Hartley, C.A., McElroy, P., McCrea, C., and McNiece, I.K. 1996. Megakaryocyte growth and development factor stimulates enhanced platelet recovery in mice after bone marrow transplantation. Blood. 88:1509-1514.

30. Fibbe, W.E., et al. 1995. Accelerated reconstitution of platelets and erythrocytes following syngeneic transplantation of bone marrow cells derived from thrombopoietin pretreated donor mice. Blood. 86:3308-3313.

31. Bolwell, B., et al. 2000. Phase 1 study of pegylated recombinant human megakaryocyte growth and development factor (PEG-rHuMGDF) in breast cancer patients after autologous peripheral blood progenitor cell transplantation (PBPC). Bone Marrow Transplant. 26:141-145.

32. Beveridge, R., et al. 1997. Randomized, double-blind, placebo-controlled trial of pegylated recombinant human megakaryocyte growth and development factor (PEG-rHuMGDF) in breast cancer patients (Pts) following autologous bone marrow transplantation (ABMT). Blood. 90(Suppl. 1):580a (Abstr.).

33. Pestina, T.I., Cleveland, J.L., Yang, C., Zambetti, G.P., and Jackson, C.W. 2001. Mpl ligand prevents lethal myelosuppression by inhibiting p53dependent apoptosis. Blood. 98:2084-2090. 